\title{
VARIABILIDADE ESPACIAL DA PRODUTIVIDADE E DA QUALIDADE DAS SEMENTES DE SOJA EM UM CAMPO DE PRODUÇÃO ${ }^{1}$
}

\author{
NILSON MATHEUS MATTIONI ${ }^{2}$, LUIS OSMAR BRAGA SCHUCH ${ }^{3}$, FRANCISCO AMARAL VILLELA $^{3}$
}

\begin{abstract}
RESUMO - Os campos de produção de sementes de soja (Glycine max L. Merrill) estão sujeitos a uma série de fatores, que influenciam na qualidade final das mesmas, sendo que esses nem sempre atuam uniformemente. O objetivo do trabalho é determinar a variabilidade espacial da produtividade e da qualidade de sementes de soja, em um campo de produção de sementes, utilizando as ferramentas da agricultura de precisão. As sementes foram colhidas seguindo um grid de 1 ponto por hectare, georeferênciado, com quatro sub-amostras, totalizando uma área colhida de $16 \mathrm{~m}^{2}$ por ponto, que após serem pesadas, estimou-se a produtividade. $\mathrm{O}$ tamanho das sementes produzidas foi determinado através do teste de retenção nas peneiras de tamanho 5,5, 6,5 e 7,5 mm. A qualidade fisiológica das sementes foi determinada pelo teste de germinação, primeira contagem, envelhecimento acelerado e emergência. Os dados apresentaram um coeficiente de variação de $9,66 \%$ para produtividade, $8,46 \%$ para germinação, $13,24 \%$ para primeira contagem, $19,01 \%$ para envelhecimento acelerado e 6,93\% para emergência. Foi verificada a dependência espacial para as variáveis estudadas, sendo que o alcance dessa dependência foi de $300 \mathrm{~m}$ para produtividade, $700 \mathrm{~m}$ para germinação e primeira contagem, $400 \mathrm{~m}$ para envelhecimento acelerado e $200 \mathrm{~m}$ para emergência. A qualidade fisiológica das sementes em um campo de produção não é uniforme, assim como a produtividade, sendo possível a estimação dessas variáveis em mapas interpolando pontos homogêneos. A variabilidade representada por mapas de interpolação é uma ferramenta da gestão de qualidade de sementes que permite a definição de áreas a serem colhidas e descartadas dentro de um campo de produção de sementes.
\end{abstract}

Termos para Indexação: produção de sementes, agricultura de precisão, germinação, vigor.

\section{SPATIAL VARIABILITY OF PRODUCTIVITY AND SEED QUALITY IN A SOYBEAN PRODUCTION FIELD}

\begin{abstract}
Seed production fields of soybean (Glycine max L. Merrill) are subject to various factors that influence the final seed quality but which do not always act uniformly. The objective of the present study was to determine the spatial variability of the productivity and quality of soybean seeds in a production field using precision farming. Seeds were collected following a grid of one point per hectare, georeferenced, with four sub-samples, totaling a harvested area of $16 \mathrm{~m}^{2}$ per point, which were used to estimate productivity after being weighed. Seed sizes were determined by the retention test in sieve sizes of $5.5,6.5$ and $7.5 \mathrm{~mm}$ and seed physiological
\end{abstract}

${ }^{1}$ Submetido em 11/09/2010. Aceito para publicação em 01/04/2011. Parte da Dissertação de Mestrado do primeiro autor apresentada à Universidade Federal de Pelotas (UFPel).

${ }^{2}$ Eng. Agr., Mestre em Ciências, UFPel, Caixa Postal: 345, CEP: $96001-$
970, Pelotas-RS.nilsonmattioni@gmail.com

${ }^{3}$ Eng. Agr., Dr., Professor Associado da UFPel, Bolsista de produtividade em pesquisa CNPq. lobs@ufpel.edu.br 
quality from germination, first count, accelerated aging and emergence tests. The data showed a coefficient of variation of $9.66 \%$ for yield, $8.46 \%$ for germination, $13.24 \%$ for first count, $19.01 \%$ for accelerated aging and $6.93 \%$ for emergence. Spatial dependence was observed for the variables, being $300 \mathrm{~m}$ for yield, $700 \mathrm{~m}$ for germination and first count, $400 \mathrm{~m}$ for accelerated aging and $200 \mathrm{~m}$ for emergence. Like productivity, seed physiological quality in a production field is not uniform, but these variables can be estimated on maps by interpolating homogeneous points. The variability represented by interpolation maps is a seed quality management tool that allows areas to be harvested or discarded within a production field.

Index terms: seed production, precision agriculture, germination vigor.

\section{INTRODUÇÃO}

A baixa qualidade das sementes de soja ocasiona severos problemas para comercialização, acarretando prejuízos com a reprovação de lotes e até mesmo campos de produção. Esse fato gera um desperdício de recursos, visto que todo o manejo da lavoura foi feito de maneira diferenciada, com altos investimentos visando garantir a produção de sementes de alta qualidade.

Segundo Lacerda (2007), os campos de produção de sementes exigem maior cuidado no controle de plantas daninhas, pureza varietal e insetos. Portanto, a eliminação de lotes por problemas de germinação causa prejuízos econômicos ao produtor. $\mathrm{O}$ autor salienta ainda que o uso de sementes de baixa qualidade pelo agricultor, muitas vezes impede o estabelecimento de adequada população de plantas e exige a realização de outra semeadura.

Estudos revelam que a investigação dos limites de produtividade contribui para identificar as variáveis do ambiente responsáveis pelo desempenho final da cultura e ressaltar em que nível cada uma delas representa limitações à expressão máxima da produtividade (Barni et al., 1995). Nesse sentido, Argenta et al. (2003) ressaltaram que a determinação do potencial de rendimento de grãos das culturas torna-se ferramenta importante para a tomada de decisões no manejo, por possibilitar a identificação dos fatores limitantes e subsidiar o dimensionamento dos impactos dos fatores restritivos ao rendimento e a definição de estratégias para superação ou minimização através do manejo adequado.

Os mapas de distribuição espacial das características do solo, mostram a grande variabilidade de ambiente que as plantas podem encontrar em uma lavoura (Mulla, 1993;
Schlindwein; Anghinoni, 2000, Amado et al., 2006). A produção vegetal está dependente de várias características genéticas e ambientais, que podem produzir características fenotípicas distintas a partir de um mesmo material genético. Frente a isso é importante conhecer se existe ou não variabilidade espacial da qualidade fisiológica das sementes, no campo de produção no momento da colheita, assim como outros fatores já estudados.

Assim, esse estudo teve o objetivo de avaliar a variabilidade espacial da produtividade e da qualidade de sementes de soja em um campo de produção, utilizando as ferramentas da agricultura de precisão.

\section{MATERIAL E MÉTODOS}

O trabalho foi realizado em um campo de produção de sementes no município de Não-Me-Toque - RS de 60,6 ha, durante a safra de soja 2008/09. Com base no mapa de contorno da área, foi elaborado um grid de amostragem, sendo os pontos amostrais espacializados de $100 \times 100$ metros, caracterizando um ponto(amostra) por hectare, utilizando software "Sistema Agropecuário CR Campeiro 6".

A semeadura da área foi realizada nos dias 17 e 18 de novembro de 2008, utilizando uma semeadora equipada com sulcador do tipo facão, e com sistema de distribuição de sementes do tipo disco perfurado. A máquina foi regulada para que distribuísse 19 sementes por metro linear, e um espaçamento entre linhas de $0,45 \mathrm{~m}$. Foi utilizado como adubação de base, uma taxa fixa de 160 $\mathrm{Kg} \mathrm{ha}^{-1}$ de adubo NPK de fórmula 2-20-20, por hectare e a cultivar A4725 RG.

Para a determinação da produtividade bem como da qualidade das sementes produzidas no campo, foi realizado 
a colheita das amostras, sendo coletadas as plantas em quatro parcelas de $1 \mathrm{~m}^{2}$ cada, sendo que as quatro subamostras formaram a amostra média do ponto. Para demarcação das áreas a serem colhidas foi considerado o ponto da malha de amostragem como ponto central, onde foi coletada uma sub-amostra e as outras três foram coletados em um raio de $10 \mathrm{~m}$ a partir do ponto central, sendo o ângulo entre cada raio de $120^{\circ}$.

As plantas colhidas foram então trilhadas em uma máquina estacionária, obtendo-se as amostras de sementes de todos os pontos da malha de amostragem.

As amostras foram submetidas ao processo de secagem artificial em estufa com circulação forçada de ar, a uma temperatura de $35^{\circ} \mathrm{C}$, até atingirem um teor de água de $12 \%$. As amostras foram pesadas em uma balança digital com precisão de $0,01 \mathrm{~g}$ e os valores transformados em $\mathrm{kg} \cdot \mathrm{ha}^{-1}$ e corrigidos para $13 \%$ de umidade.

Para determinar o tamanho das sementes produzidas, as amostras foram submetidas ao teste de retenção em peneiras. Para a realização do teste dotou-se de três peneiras de furos redondos de malha 7,5; 6,5 e 5,5 mm e mais uma sem perfurações (fundo cego). As frações retidas em cada peneira foram então retiradas e pesadas separadamente em uma balança com precisão de $0,01 \mathrm{~g}$. Os resultados foram transformados em $\mathrm{kg} \cdot \mathrm{ha}^{-1} \mathrm{e}$ corrigidos para $13 \%$ de umidade, representando o percentual de cada fração de sementes em relação a produtividade.

Para a determinação da qualidade fisiológica das sementes, foram realizados os seguintes testes:

Teste de germinação - realizado em quatro repetições de 50 sementes para cada amostra, colocadas para germinar em substrato de papel de germinação ("germitest"), previamente umedecido em água utilizando-se 2,5 vezes a massa do papel seco. Posteriormente, as sementes foram acondicionadas em um germinador, na temperatura constante de $25{ }^{\circ} \mathrm{C}$. As contagens de germinação foram efetuadas no quinto e no sétimo dia após a instalação do teste, efetuadas segundo critérios estabelecidos pelas Regras para Análise de Sementes- RAS (Brasil, 1992).

Primeira contagem - realizado conjuntamente com o teste de germinação, onde se determinou o número de plântulas normais no quinto dia após a instalação do teste de germinação.

Envelhecimento acelerado - foram utilizadas caixas plásticas do tipo gerbox como compartimento individual. No interior dessas caixas foi feita a adição de $40 \mathrm{~mL}$ de água, conforme Panobianco e Marcos Filho (1998). As sementes foram colocadas em camada única sobre uma tela acondicionada dentro da caixa gerbox, e mantidas a $41{ }^{\circ} \mathrm{C}$ durante 48 horas. Posteriormente realizou-se o teste de germinação, realizando uma única contagem no quinto dia.

Emergência de plântulas - foram semeadas quatro sub-amostras de 20 sementes em bandejas plásticas com vermiculita previamente umidecida. As bandejas foram mantidas em ambiente fechado com temperatura controlada a $20{ }^{\circ} \mathrm{C}$. A contagem das plântulas normais emergidas foi realizada ao quinto dia após a semeadura (Brasil, 1992).

Os resultados das análises de produtividade, teste de retenção em peneiras e da qualidade fisiológica das sementes foram utilizados para a elaboração de modelos digitais (mapas) através do software "Sistema Agropecuário CR - Campeiro 6" (Giotto et. al., 2004).

O método geoestatístico de interpolação utilizado na elaboração dos modelos digitais foi a krigagem, com raio máximo de pesquisa de 150 metros.

\section{RESULTADOS E DISCUSSÃO}

O ciclo da cultura foi de 111 dias entre a emergência e a maturidade fisiológica e 115 dias entre a emergência e a colheita das sementes, sendo essas colhidas com umidade média de $15,5 \%$.

A área apresentou elevada produtividade, com uma média de $3.548,47 \mathrm{~kg} \cdot \mathrm{ha}^{-1}$. Os valores variaram entre mínimos e máximos de 2.789,43 a 4.280,81 kg.ha-1, uma amplitude de 1491,38 kg.ha-1 ${ }^{-1}$, porém um CV baixo $(9,66 \%)$, sendo a distribuição espacial ilustrada na Figura 1. Rosa Filho et al. (2009), estudaram a variabilidade da produtividade de grãos de soja em função de atributos do solo, diagnosticaram uma variabilidade média expressa por um coeficiente de variação de $14 \%$, porém, Johann et al. (2004), por sua vez, encontraram uma alta variabilidade expresso pelo $\mathrm{CV}$ de $23 \%$.

O desenvolvimento e a produção das culturas, segundo Abreu et al. (2003) e Silva et al. (2003), apresentam variabilidade espacial de acordo com o comportamento das propriedades físicas ou químicas do solo mais limitantes ao desenvolvimento da planta, sendo fatores importantes de análise para o planejamento e a avaliação de experimentos e de lavouras comerciais. Porém essas variáveis não ocorrem uniformemente no solo podendo ser uma possível causa de variabilidade da produtividade. 

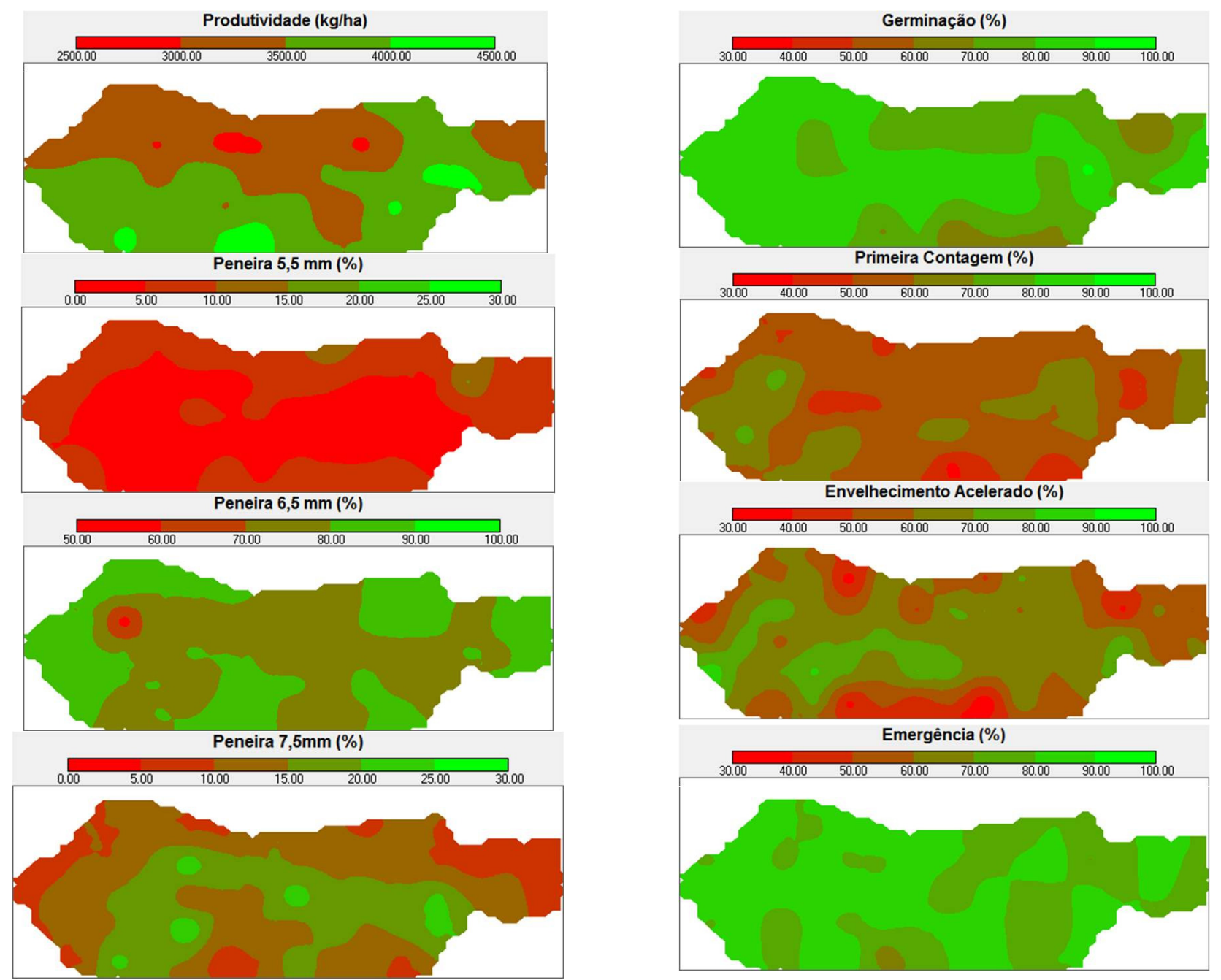

FIGURA 1. Mapas de variabilidade espacial da produtividade, tamanho de semente (peneira 5,5; 6,5 e 7,5 mm), germinação, primeira contagem, envelhecimento acelerado e emergência de soja na safra 2008/09.

O mapa de produtividade é uma importante ferramenta na tomada de decisão e análise de desempenho agrícola em nível de propriedade (Amado et al., 2007). Molin (2002) e Milani et al. (2006), destacam ainda, que o mapa de produtividade é a alternativa mais completa e moderna para gerenciar a variabilidade espacial de lavouras comerciais, orientando práticas de manejo.

Nos mapas do tamanho de semente (Figura 1) é possível observar a variabilidade espacial da percentagem de sementes, em cada peneira avaliada. Observa-se também um CV alto para as peneiras 5.5 e $7.5 \mathrm{~mm},(46,72 \%$ e $31,23 \%$, respectivamente) e baixo para a peneira $6.5 \mathrm{~mm}$ $(5,81 \%)$. Pode-se dizer que houve uma maior produção das sementes classificadas na peneira $6.5(79,2 \%)$ tendo, porém, zonas produzindo $54 \%$ e outras $85 \%$ das sementes com esse tamanho.

Pode-se inferir que o tamanho médio das sementes no campo de produção não apresenta variabilidade considerável, porém os valores extremos mostram-se mais sensíveis as mudanças do ambiente, o que é evidenciado pela sua alta variabilidade.

As sementes produzidas na área objeto desse estudo apresentaram uma germinação média de $80 \%$, com os valores variando entre 61 e $93 \%$. Analisando os dados relativos ao teste de germinação, é possível observar um CV baixo (8,46\%). Estudo realizado por Mondo (2008) destacou dados semelhantes, onde obteve-se valor médio de germinação de $88 \%$, e valores máximo e mínimo de 98 e $64 \%$, respectivamente, além de um CV baixo $(7,6 \%)$.

Os testes de primeira contagem e envelhecimento 
acelerado apresentaram coeficientes de variação de 13,24\% e $19,01 \%$, respectivamente, mostrando que o vigor apresentase mais sensível às variações ambientais, quando comparado com a viabilidade das sementes. Segundo Delouche (2002), durante o processo de deterioração das sementes, a perda da capacidade de germinar é o último processo antes da morte da semente, enquanto que os testes de vigor, se baseiam em eventos que acontecem anteriormente. $\mathrm{O}$ teste da primeira contagem, por exemplo, está baseado na velocidade de germinação, enquanto que o teste de envelhecimento acelerado está baseado na capacidade de armazenamento, eventos esses que segundo Delouche (1969), antecedem a perda da viabilidade das sementes.

Os testes de vigor apresentaram valores mínimos de $37 \%$ e $30 \%$, máximos de $75 \%$ e $89 \%$ e médios de $57 \%$ e $62 \%$ para os testes de primeira contagem e envelhecimento acelerado, respectivamente. A distribuição dessas regiões dentro da área pode ser observada na Figura 1, onde é possível fazer o ranqueamento das diferentes regiões da área pelo vigor das sementes.

Assim sendo, os testes de vigor permitem um melhor diagnóstico da qualidade fisiológica das sementes, pois apresentam uma maior sensibilidade no diagnóstico da sua deterioração, possibilitando com o estudo da variabilidade espacial e mapeamento, distinguir com maior precisão as regiões dentro do campo de produção que possuem sementes com qualidade superior.

A viabilidade das sementes estimada pelo teste de emergênciadeplântulas foi semelhanteaotestedegerminação, como ilustrado pela Figura 1, sendo $65 \%, 90 \%$ e $89 \%$, os valores mínimos, máximos e médios respectivamente.

Mesmo com todas as ferramentas disponíveis para o manejo da lavoura, buscando máximas produtividades e no caso das sementes alta qualidade fisiológica, isso nem sempre é possível. Estudos realizados por Costa et al. (2003) têm mostrado que apesar de toda tecnologia disponível, a quantidade de sementes provenientes de algumas regiões tem sido severamente comprometida em função dos elevados índices de deterioração por umidade, de lesões por percevejos, por quebras, ruptura de tegumento e danos mecânicos.

Observando o mapa da variabilidade espacial do teste de germinação (Figura 1), é possível localizar as zonas onde as sementes apresentam qualidade fisiológica inferior. Levando-se em conta que o padrão mínimo de germinação para comercialização de sementes de soja é $80 \%$, é possível distinguir, as zonas que apresentam qualidade fisiológica satisfatória, o que pode ser uma informação importante, para definir o destino dessas sementes na Unidade de Beneficiamento de Sementes (UBS), ou até mesmo proceder o descarte parcial do campo eliminando zonas de qualidade fisiológica inferior.

A análise dos semivariogramas (Figura 2) permite inferir que o grid de amostragem de um ponto por hectare, foi eficiente para obtenção de amostras representativas e estimação da qualidade das sementes e produtividade (Tabela 1). Os alcances obtidos para as variáveis germinação, primeira contagem, envelhecimento acelerado, emergência e produtividade, foram de 700, 700, 400, 200 e $300 \mathrm{~m}$ respectivamente. Segundo Vieira (2000), o alcance é a distância limite de dependência espacial, onde amostras separadas por distâncias menores que o seu valor, são correlacionadas umas às outras, o que permite que se faça interpolações para espaçamentos menores do que os amostrados.

TABELA 1. Valores máximos, médios, mínimos e coeficiente de variação (CV) da produtividade, porcentagem de sementes peneira $5,5 \mathrm{~mm}$, porcentagem de sementes peneira $6,5 \mathrm{~mm}$, porcentagem de sementes peneira 7,5mm, germinação, primeira contagem, envelhecimento acelerado e emergência de plântulas de sementes de soja, safra 2008/09.

\begin{tabular}{lcccc}
\hline & Máximo & Mínimo & Médio & CV $(\%)$ \\
\hline Produtividade (kg/ha) & 4281 & 2789 & 3548 & 9,6 \\
Peneira 5.5 (\%) & 15 & 2 & 5,3 & 31,23 \\
Peneira 6.5 (\%) & 85 & 54 & 79,2 & 5,8 \\
Peneira 7.5 (\%) & 24 & 7 & 13,7 & 46,72 \\
Germinação (\%) & 93 & 61 & 80 & 8,4 \\
Primeira contagem (\%) & 57 & 37 & 75 & 13,24 \\
Envelhecimento acelerado (\%) & 89 & 30 & 62 & 19,01 \\
Emergência (\%) & 90 & 65 & 80 & 6,95 \\
\hline
\end{tabular}




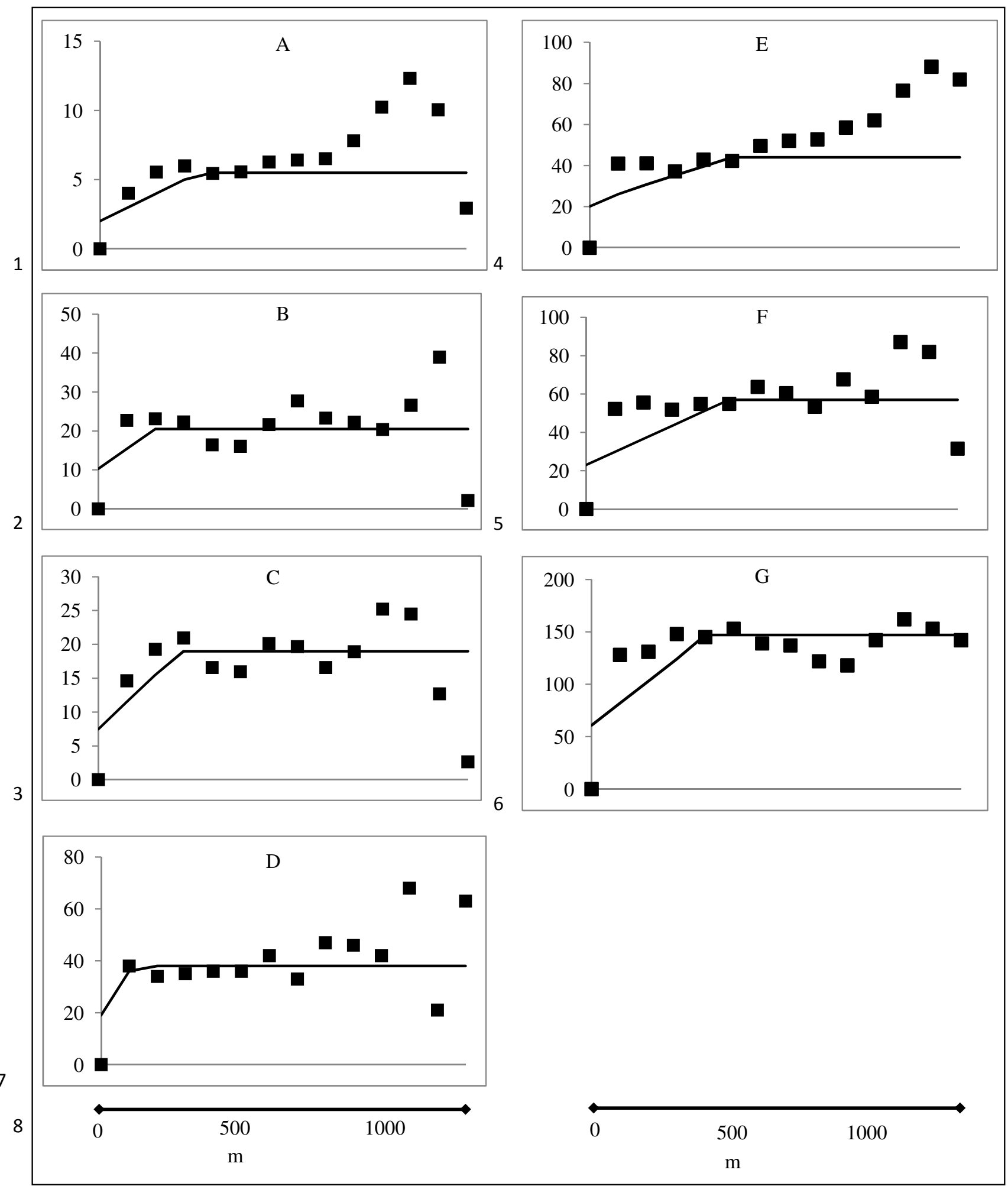

FIGURA 2. Semivariogramas das variáveis produtividade, tamanho de sementes (peneiras 5,$5 ; 6,5$ e 7,5 mm), germinação, primeira contagem, envelhecimento acelerado e emergência 
Assim sendo, com a utilização do grid de 1 ponto por hectare, com uma distância de $100 \mathrm{~m}$ entre cada ponto, permitem a geração de mapas de interpolação, que permitem inferências da variabilidade espacial dos fatores estudados.

\section{CONCLUSÕES}

A produtividade da cultura da soja é desuniforme nos campos de produção de sementes, com diferença de

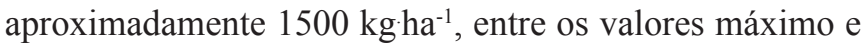
mínimo. Existe também uma variabilidade alta na produção de sementes maiores e menores, sendo as sementes de tamanho médio pouco afetadas.

A qualidade fisiológica de sementes de soja não é uniforme nos campos de produção, sendo que o vigor, demonstra-se mais sensível e, portanto, com maior variabilidade quando comparado com a germinação.

A variabilidade representada por mapas de interpolação é uma ferramenta da gestão de qualidade de sementes que permite a definição de áreas a serem colhidas e descartadas dentro de um campo de produção de sementes.

\section{AGRADECIMENTOS}

Agradecemos ao Programa de Pós-Graduação em Ciência e Tecnologia de Sementes da Universidade Federal de Pelotas (UFPel) pela oportunidade de realização deste trabalho e ao Conselho Nacional de Desenvolvimento Científico e Tecnológico (CNPq), pela bolsa de estudo concedida ao primeiro autor, durante o curso de Mestrado em Ciências.

\section{REFERÊNCIAS}

ABREU, S.L.; REICHERT, J.M.; SILVA, V.R.; REINERT, D.J.; BLUME, E. Variabilidade espacial de propriedades físicohídricas do solo e da produtividade e qualidade de grãos de trigo agroecológico em argissolo franco arenoso sob plantio direto. Ciência Rural, v.33, n.2, p.275-282, 2003.

AMADO, T.J.C.; BELLÉ, G.L.; DELLAMEA, R.B.C.; PES, L.Z.; FULBER, R.; PIZZUTI, L.; SCHENATO, R.B.; LEMAINSKI, C.L. Projeto Aquarius-Cotrijal: pólo de agricultura de precisão Revista Plantio Direto, v.91, n, 2006.

AMADO, T.J.C.; PERES, R.B.; COSTA, J.A.; NICOLOSO, R.S.; TEIXEIRA, T.G. A safra recorde analisada pelos mapas de rendimento no RS. Revista Plantio Direto, n.101, p.18-123, 2007.

ARGENTA, G.; SANGOI, G.; SILVA, P.R.F. da.; RAMPAZZO, C.; GRACIETTI, L.C.; STRIEDER, M. FORSTHOFER, E.L.; SUHRE, E. Potencial de rendimento de grãos de milho em dois ambientes e cinco sistemas de produção. Scientia Agrícola, v.4, n.1/2, p.27-34, 2003.

BARNI, N.A.; BERLATO, M.A.; BERGAMASCHI, H.; RIBOLDI, J. Rendimento máximo do girassol com base na radiação solar e temperatura: II. produção de fitomassa e rendimento de grãos. Pesquisa Agropecuária Gaúcha, v.1, n.2, p.201-216, 1995b.

BRASIL. Ministério da Agricultura e da Reforma Agrária. Regras para análise de sementes. Secretaria Nacional de Defesa Agropecuária. Departamento Nacional de Produção Vegetal. Coordenação de Laboratório Vegetal. Brasília, DF, 1992, 365p.

COSTA, N.P. da; MESQUITA, C. de M.; MAURINA, A.C.; FRANÇA NETO, J. de B; KRZYZANOWSKI, F. C., HENNING, A.A. Qualidade fisiológica, física e sanitária de sementes de soja produzidas no Brasil. Revista Brasileira de Sementes, v.25, n.1, p.128-132, 2003.

DELOUCHE, J.C. Germinação, deterioração e vigor da semente. Seed News, v.6, n.6, p.24-31, 2002.

DELOUCHE, J.C. Planting seed quality. State College, Mississippi, Agricultural Experiment Station. Mississippi State University, 1969 (Journal Paper, 1721).

GIOTTO, L; ROBAINA, A.D.; SULZBACH, L.A Agricultura de precisão com o sistema CR campeiro 5, Manual do Usuário, 2004. 330p.

JOHANN, J.A.; URIBE-OPAZO, M.A.; SOUZA, E.G.; ROCHA, J.V. Variabilidade espacial dos atributos físicos do solo e da produtividade em um Latossolo Bruno Distrófico da região de Cascavel, PR. Revista Brasileira de Engenharia Agricola e Ambiental, v.8, n.2/3, 212219, 2004.

LACERDA, A.L.S. Fatores que afetam a maturação e qualidade fisiológica das sementes de soja (Glycine max L.). 2007. Artigo em Hypertexto. Disponível em: $<$ http:// www.infobibos.com/Artigos/2007_3/maturacao/index. htm>. Acesso em: 9/2/2010

MILANI, L.; SOUZA, E.G. de; URIBE-OPAZO, M.A.; GABRIEL FILHO, A.; JOHANN, J.A.; PEREIRA, J.O. Unidades de manejo a partir de dados de produtividade. Acta Scientiarum Agronomy, v.28, n.4, p.591-598, 2006. 
MOLIN, J.P. Definição de unidades de manejo a partir de mapas de produtividade. Engenharia Agrícola, v.22, n.1, p.83-92, 2002.

MONDO, V.H.V.; GOMES JUNIOR, F.G.; PINTO, T.L.F.; MARCHI, J.L.; MOTOMIYA, A.V.A.; MOLIN, J.P.; CICERO, S.M. Variabilidade espacial da qualidade das sementes em uma área de produção de soja. In: CONGRESSO BRASILEIRO DE AGRICULTURA DE PRECISÃO, 2008, Piracicaba- SP. Anais... Piracicaba: Esalq, 2008. p.158-169.

MULLA, D.J. Mapping and managing spatial patterns in soil fertility and crop yield. In: ROBERt, P.C.; RUST, R.H.; LARSON. W.E. (Ed). Soil specific crop management. Madison: ASA, 1993. p.15-26.

PANOBIANCO, M.; MARCOS FILHO, J. Comparação entre métodos para avaliação da qualidade fisiológica de sementes de pimentão. Revista Brasileira de Sementes, v.20, n.2, p.306-310, 1998.
ROSAFILHO, G.; CARVALHO, M.deP.E; ANDREOTTI, M.; MONTANARI, R.; BINOTTI, F.F. da S.; GIOIA, M.T. Variabilidade da produtividade da soja em função de atributos físicos de um latossolo vermelho distroférrico sob plantio direto. Revista Brasileira de Ciência do Solo. v.33, p.283-293, 2009.

SCHLINDWEIN, J.A.; ANGHINONI, I. Variabilidade horizontal de atributos de fertilidade e amostragem do solo no sistema plantio direto. Revista Brasileira Ciência do Solo, v.24, n.1, 85-91, 2000.

SILVA, V.R.; REICHERT, J.M.; STORCK, L.; FEIJO, S. Variabilidade espacial das características químicas do solo e produtividade de milho em um Argissolo VermelhoAmarelo distrófico arênico. Revista Brasileira Ciência do Solo, v.27, n.6, p.399- 406, 2003.

VIEIRA, S.R. Geoestatistica em estudos de variabilidade espacial do solo. In: Tópicos em Ciência do Solo I. Viçosa, MG.: Sociedade Brasileira de Ciência do Solo. 2000. 352p. 